\title{
A Family Doctor's Clinical-Epidemiological Diary in Spain of the Times of Covid-19 Chapter 2: March 16-31: The Big Bang
}

\section{Turabian JL*}

Specialist in Family and Community Medicine, Regional Health Service of Castilla la Mancha (SESCAM), Spain

*Corresponding author: Jose Luis Turabian, Health Center Santa Maria de Benquerencia. Regional Health Service of Castilla la Mancha (SESCAM), Toledo, Spain, E-mail: jturabianf@

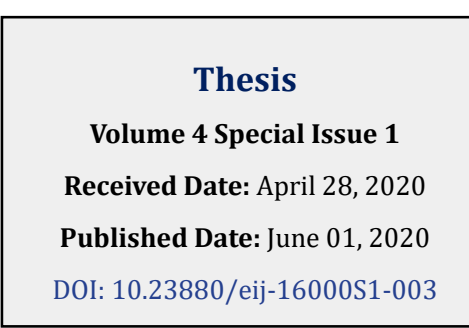
hotmail.com

\section{Abstract}

Since outbreak of coronavirus disease 2019 (COVID-19) started in late December in China, there are many scientific articles that have been published about this virus. However, data and qualitative research are lacking. In this sense, a diary written by a family doctor in Spain during the COVID-19 pandemic is presented, which can constitute a qualitative clinical-epidemiological database and a tool to estimate effects. This is "chapter 2 (March 16-31): The Big Bang." The number of respiratory infections and contacts with patients shot up exponentially (between 20-50\% of consultations), but by not doing PCR for diagnosis, this figure mixes real cases of COVID-19 with other etiologies. Even the great anxiety generated in the population can confuse symptoms. Almost all of the consultations were made by telephone. Several nearby healthcare professionals became ill. The COVID-19 bears the mark of true death, very different from its logical and abstract possibility.

Keywords: Coronavirus; COVID-19; SARS-CoV-2; General Practice; Epidemiology; Reflection; Diary Method; Professional Self-Insight; Qualitative Research

Abbreviations: PCR: Polymerase Chain Reaction.

\section{Introduction}

Since the COVID-19 infection began in late December 2019 in China, there are many scientific articles that have been published about this virus, the people affected, health teams and public health tools to combat it, which gives origin of a large volume of information. If a simple search in PubMed is carried out with the term COVID-19, more than 6,000 results appear which can give an idea of the intensity of effort that has been put in place to understand and tackle the pandemic [1].

The family doctor / General Practitioner, for clinical and epidemiological purposes, resemble the naturalist. The naturalist carries a "field notebook" (the "clinical record").
This is the main tool of every naturalist where he/she record his discoveries and impressions. Without it he/she would be likely to return home and fail to remember even half of what he had seen. The naturalist should always note the date, the place, the time and the weather. There may also be other useful data, for example, if it has been raining steadily several weeks, whether the autumn frost have begun, if the field is suffering a drought, or any other thing that he deems to be important or out of the ordinary and that could determine the behavior or appearance of some of the creatures. In addition, the naturalist looks for footprints and tracks. We can call evidence or "footprints" or "traces" to that kind of initial information. This evidence can be in the form of scars, signs, or it may relate to epidemiological data $[2,3]$.

In this context, despite the excess of quantitative biomedical information that is sometimes contradictory or 


\section{Epidemiology International Journal}

confusing, qualitative data and research are lacking. In this way, a diary written by a family doctor in Spain during the COVID-19 pandemic is presented, which can constitute a qualitative clinical-epidemiological database and a tool to estimate effects.

Here the second part of the "diary of the times of the COVID-19" is presented, where the author, family doctor of a health center in Toledo (Spain) writes the narration of the personal experiences that he is living, data and facts, and some meditations about the situation that affect him, and where he express his feelings in reference to the brief time frame of the "COVID-19 era" [4,5].

\section{Method}

The diary method is a qualitative (and quantitative) study tool [6]. The methodology of this diary has been previously described [7]. In the investigation method of the diary, the question of the representativeness of the sample can be raised; How "typical" was the participant's behavior? Will the "typical" activity be found if the sample is larger? Is the activity to be collected subject to fluctuations? This topic depends in part on the degree of generalization required. Obviously, the places and dates that the diary is kept must be carefully decided. The diary technique, used with due precautions, can achieve results that are probably close to the "true" frequency of a given period, but a study that compares it is necessary to be sure [8].

This Diary will have the following chapters, that although they are separate texts for easy reading, and represent three different stages of the COVID-19 epidemic, they are a single "times of COVID-19" story:

1) Chapter 1: February 24 To March 15: Is Covid-19 A Lottery? [7]

2) Chapter 2: March 16-31: The Big Bang

3) Chapter 3: April 1 To 31: Some Good News And Hope By The End

\section{Chapter 2: March 16-31: The Big Bang}

\section{March 16, 2020}

In Castilla La Mancha there are 567 cases and 17 deaths. In Spain: 9407 infected 335 dead and 530 recovered.

Triage begins at the Health Center. Patients are assessed the entrance of the center by a nurse and the respiratory patients are conducted to a separate area. If a patient has mild symptoms, they are asked to stay home; for moderate symptoms he or she is sent to a separate area and the patient is given a mask; If the patient is severe, he or she are not even visited, and is sent directly to the hospital. All patients with respiratory symptoms are treated as persons under investigation, for whom isolation precautions are required; In addition, for these patients, the use of personal protective equipment by doctor is required... However, there are only a couple of protective equipment in the center... But I advise that all consultations are by phone. Some patients do not keep their previous appointments. Scheduled visits to chronic patients are cancelled. The waiting rooms are almost empty. All non-urgent activity has been suspended. In the current community transmission situation, patients are no longer systematically asked about their travel history to countries with high transmission; in theory, any patient with fever, cough and dyspnea should be evaluated immediately for COVID-19, using the COVID-19 polymerase chain reaction (PCR). But this test is not being done except in hospital. PCR is not accessible to family doctors. In this way, "every respiratory infection becomes a possible case of COVID", which will imply isolation, contact management, and sick leave. Furthermore, great anxiety is generated in patients ... And in me: I don't know what I'm dealing with $[9,10]$.

I am wearing a mask and gloves ... I'm fine, but I feel worried ... It seems to me that there will be no way to avoid being infected; And without counting the air transmission, the permanence of the virus on surfaces ... Although I try to do everything well, it is almost impossible ... Everything will be a matter of luck $[11,12]$.

\section{March 17}

662 cases and 23 deaths in Castilla La Mancha.

A much-loved medical partner is admitted to the hospital by COVID-19. I am very concerned about his clinical condition. She has made a cytokine storm...

\section{5 patients}

A 33-year-old man who comes personally for a painful shoulder! I explain the inadequacies of his visit... The vast majority of consultations are by phone: some repetition of drug prescriptions for known chronic diseases, some consults for new problems, and several consultations for anxiety due to COVID-19 are gathered.

A 65-year-old man with itching, 43-year-old woman with idiopathic temporal epilepsy, 71-year-old man with severe tearing, 61-year-old man years with low back pain, 75-year-old woman for repetition of hypertension medication, 22-year-old woman requests medication after cosmetic breast surgery, 74-year-old woman for allergic rhinitis medication, a 92-year-old woman who fell a few days ago and was attended in the emergency department, with polyconusions, she now reports that she is in a lot of 


\section{Epidemiology International Journal}

general pain that does not improve with acetaminophen, a 33-year-old woman who simply asks for the prescription of " 2 boxes of ibuprofen to have at home"!, a 21-year-old man with extrinsic allergic asthma, a 73-year-old man with abdominal pain in the right abdomen and nausea without vomiting, and accompanying headache without fever, A 70-year-old man with hypertension, a 32-year-old woman with allergic rhinitis, a 42-year-old woman who "may be pregnant", a 64-year-old woman anxious about her work situation and about the care of her sick husband... and due to the coronavirus... A 55-year-old man who requests a report of his risks in the case of an intercurrent disease such as a viral infection and he asks for a sick leave from work, a 46-yearold man with anxiety to fear getting a coronavirus and "he is considered as a risk group for having been operated on for pleural disease."

\section{PERCENTAGE OF PATIENTS WITH RESPIRATORY INFECTION WITH RESPECT TO THE TOTAL OF PATIENTS CARED}

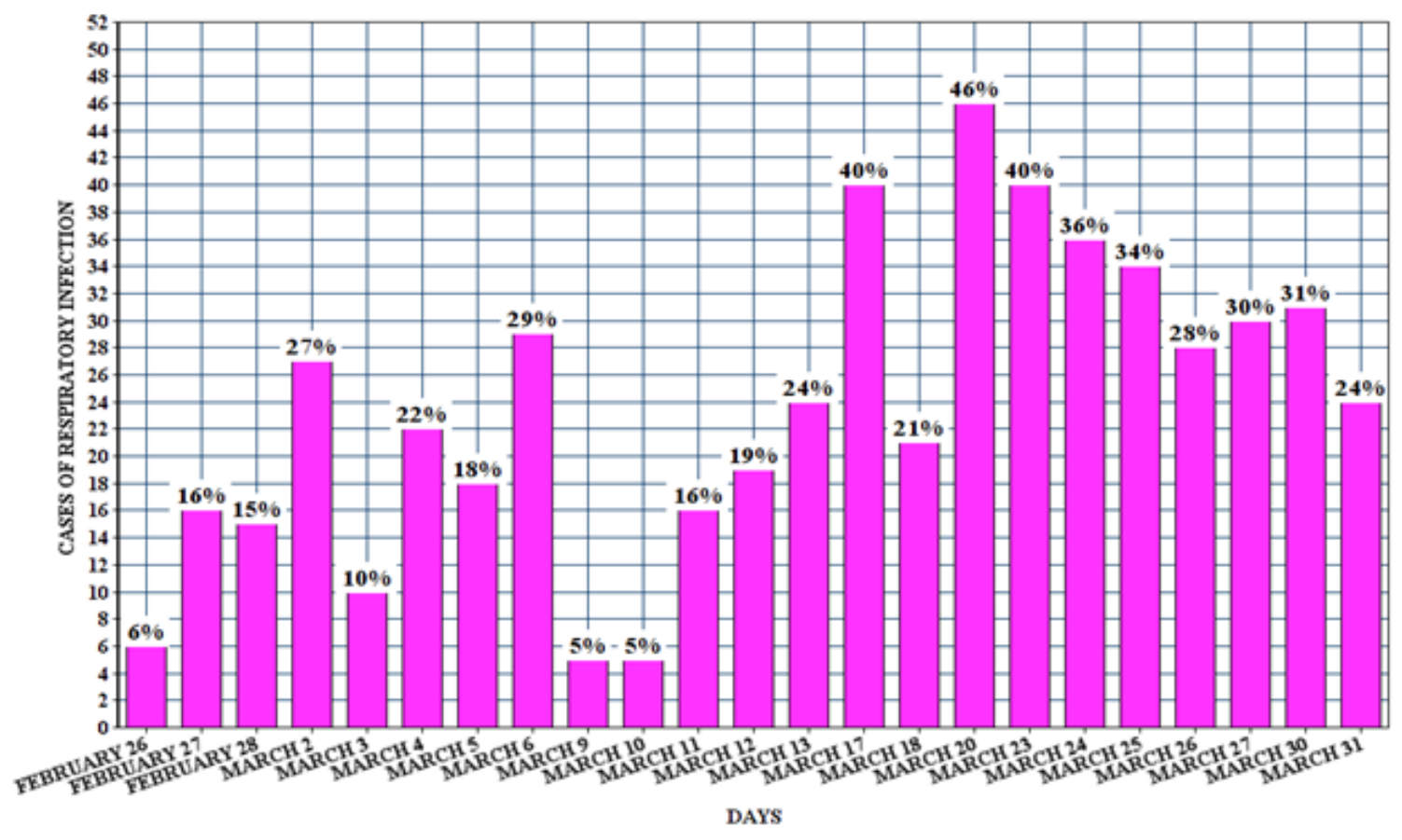

Figure 1: Cases of Respiratory Infection, Contacts and Monitoring Of Both Regarding the Total of Patients Cared.

And respiratory infections: a 45-year-old woman who says that "she caught from her daughter who had a cold a couple of days ago"; she is coughing without fever, a 34-yearold man with a cough and fever; he works at a gas station, a 72-year-old woman with general malaise, cough without respiratory distress and without fever or low fever, a 30-yearold woman with a slight cough, a 50-year-old man with a fever of $38^{\circ}$ since yesterday and "dry throat"; his wife is a nurse, a 36-year-old man with a cough and fever, a 21-year-old man who has been coughing for 3 days with expectoration, mild odynophagia and low-grade fever, a 49-year-old man attended by his company's doctor for rhinorrhea and mild cough, who advised him to be off work, a 47-year-old woman who has had asthenia, anorexia, nausea and doubtful diarrhea for 6 days; no fever or minimal fever; without cough, a 29-year-old male who works in construction with a dusty and gypsum environment... and worsened his asthma with more cough and dyspnea; he is concerned about COVID-19,

I should seek a rapid diagnosis of patients suspected of having COVID-19. But I don't have the PCR. Probably more than $80 \%$ of people infected with COVID-19 remain undiagnosed. This action could preserve the function of the health system. Is the diagnosis with PCR what causes the differences that can be observed regarding the mortality rate of COVID-19: 1\% in South Korea, 8\% in Italy and 4\% in Spain as of March 17, 2020? [13]. 


\section{Epidemiology International Journal}

I am called from the Epidemiology Service: a 66-yearold man, patient of mine although I have not treated him now, is admitted to the hospital for confirmed COVID-19 pneumonia. He entered on March 14 with fever, cough and dyspnea; he denied contact with a confirmed case, but reported having been in Madrid. Chest x-ray with infiltrate in the right upper lobe and doubtful in the right lower lobe; Ac IgM anti Chlamydia pneumoniae: Negative; Ac IgM anti Mycoplasma pneumoniae: Negative; Ag-Ac HIV: Negative; Ag Pneumococcus: Negative. He is being treated with azithromycin, ceftriazone, lopinavir and hydroxychloroquine.

When I reviewing his medical history, I observed he had another hospital admission on February 12, 2020 for fever with dyspnea of minimal effort, cough and green expectoration. His chest X-ray revealed multiple patchy and confluent lung opacities, poorly defined, especially in the right hemithorax. He was diagnosed with bilateral community-acquired pneumonia with partial respiratory failure, and suspected influenza. He was treated with Ceftriaxone, Levofloxacin, Oseltamivir and oxygen therapy. The pneumococcal and legionella urine antigen were negative, but infection with influenza $A$ and $B$ viruses could not be ruled out due to poor transport of the sample.

Could that first pneumonia 40 days ago be COVID-19? Could the COVID-19 have arrived in Spain and Castilla La Mancha months ago? And in this case, immunity would be very short-lived... Perhaps it could be studied if there was a strange increase in potentially suspicious pneumonia in the past few months. In that case it could be assumed that the infection went unnoticed for months [14].

\section{March 18th}

In Castilla La Mancha 881 cases and 42 deceased. In Spain 13,910 infected, 623 dead and 1,081 recovered

24 patients by phone; there are many inquiries of anxiety about COVID-19.

The guidelines of not carrying out the diagnostic test for COVID-19 in people with a clinical picture of mild acute respiratory infection in the community, as happens on this date of March 18, 2020 in Spain, in turn cause an "epidemic of anxiety" in the community: in patients called "possible cases"(which are actually "al "cases with mild acute respiratory infection, since there is no diagnostic test); in caregivers, relatives and other co-workers, school, neighborhood contacts, etc.; and in myself as a doctor who treats them... Perhaps the COVID-19 pandemic will drag behind it another pandemic of mental illness $[9,15,16]$.

Also quite a few queries that bring sick leave. The non-
PCR situation in respiratory infections originating other "epidemic of sick leave", in the possible cases, the possible contacts and in the asymptomatic people but distressed to be in situation of some type of work or family risk, or simply for anxiety [5].

A 40-year-old woman had an intermittent fever last Monday, a 53-year-old woman with a cough and headache; she works at the hospital, a 29-year-old man who is scared because of having a low-grade fever yesterday, a 56-yearold woman with a cough, malaise and low-grade fever, and a woman with a cough and odynophagia.

How many COVID-19 patients should I expect to have on my patient list? Because diagnostic tests are not being carried out, on the one hand, all patients with respiratory symptoms are being considered as COVID-19; and on the other hand, the only way to approximate the number of possible infected is using mathematical models, basically based on the number of deaths [17-20]. Applying these estimates to the Castilla-La Mancha region (with 567 official cases and 17 deaths), there would be 13,600 real cases, and regarding my list of patients (2000 patients> 14 years old) I will have approximately 1 "official" case and 26 hidden real cases [9].

\section{March 20}

Yesterday 19 was a holiday (San José; my saint!).

1,423 "detected" cases, and 84 deaths in Castilla La Mancha. In Spain 9,980 infected, 1,002 dead and 1,588 recovered.

22 patients; all were telephone inquiries.

Respiratory cases: 39-year-old man who cares for his mother with possible respiratory infection COVID-19, 40-year-old woman with diarrhoea, anxiety, cough and fever, a 39-year-old man with odynophagia without fever, a 69-year-old man with odynophagia without fever or dyspnea and slight cough, a 40-year-old woman with fever, headache and anxiety; she works in the hospital "where there are several cases", a 33-year-old man with a fever and a slight cough, a 37-year-old woman with pharyngitis and a slight cough; "she fears that his co-workers will be alarmed when they hear her cough", a 24-year-old woman contacted a coworker in Madrid with a cough and fever without a PCR test, a 37-year-old woman with a cough for 20 days without other symptoms, and a 46-year-old woman with cough and discomfort with low-grade fever.

It seems to me that I am entering the explosion phase of the epidemic: there is an exponential number of patients with symptoms...: it is the Big Bang. I believe that the risk 


\section{Epidemiology International Journal}

of transmission was underestimated, perhaps due to ignorance..., but at the same time, other countries were implementing measures to reduce the risk and it seems that they are succeeding! Furthermore, there is a lack of means... even within the system to avoid transmission within health centers and hospitals.

At the Health Center, a computer system of "common respiratory patient agenda" is being used, so that any doctor can attend to them regardless of who their general practitioner is. The companions tell me: "The common agenda... is a system for that when we will fall ill another companion can continue caring for our patients..., we will fall like flies..."

I'm worried. I am 64 years old... I had other serious illnesses but I did not see the imminent danger on life that I see now... I am afraid of infecting my wife... we sleep separated; I try to keep a certain distance. We did not kiss... At first I was afraid of catching and getting sick... But perhaps that would imply that I would have to isolate myself at home for 14 days and nothing more... But now I am seeing how my age patients get worse after two weeks... Now I am afraid of catching and dying. I have never felt this before... COVID-19 bears the mark of true death, very different from its logical and abstract possibility.

\section{3 of March}

I have known that a family doctor who worked at another nearby health center has died. He was my age! He had bilateral pneumonia... The afternoon nurse in the office next to mine is positive... She is at home. The patients ask me how I am, and they advise me to take care of myself. It is a novelty; until now it was I who asked and advised them.

Of course, in Spain the explosion of cases was unexpected... We lack foresight, intelligence and modesty. I am inside turbulence; inside the Big Bang.

Today March 23: 2,078 cases and 145 deceased in Castilla La Mancha. In Spain 33,089 infected, 2,184 dead and 3,355 recovered

43 patients; among respiratory cases and contacts with respiratory cases, I attended 16 patients. All inquiries are by telephone. Except for the warfarin controls, for which the patients come, and are only 3 or 4 people, the waiting room is empty. The large empty waiting room feels cold and ghostly. The nurses with mask, gloves, and gown... seem like ghostly visions... Although they were the ones who made their own caps and disposable gowns! Not enough protection material yet. The demand that seemed uncontrollable has spontaneously softened. Almost half of the consultations are for respiratory symptoms. The rest of the diseases seem to be hibernated...

I'm prescribing over the phone! Also I am prescribing antibiotics. I had never done this before! I choose azithromycin... But, I can't find criteria based on evidence. I certainly don't feel good acting like this. Antibiotics should be reserved for patients suspected of having concomitant bacterial or fungal infections, or for Immunosuppressed patients are at high risk of secondary infection, or for pediatric cases of COVID-19 where underlying coinfection may be more common.

\section{March 25th}

Today are 2,780 cases and 263 deceased in Castilla La Mancha.

38 patients; some patient or their relatives send photos of skin lesions to the mobile phone of the nurse who works in my office... The mother of a 14-year-old man sends one of them; she says that "she has looked at photos on the Internet and one of them looks like his son's injury." The truth is that I fear that this system will become general...

There have been 13 cases of respiratory clinic, or contacts or follow-up of these patients. One of them is a 52-year-old female nurse who was discharged from the hospital with the diagnosis of mild pneumonia by COVID-19; she entered with fever of up to $38.5^{\circ} \mathrm{C}$, general malaise, anorexia, asthenia, very mild dry cough; chest x-ray: alveolar consolidation in the left lower lobe, with isolated adjacent patched focal infiltrates; she was treated with hydroxychloroquine, azithromycin, and ceftriaxone.

\section{March 26th}

In Castilla La Mancha 3,383 cases and 316 deaths.

The nurse who works in my office starts with diarrhoea and cough and goes home... She hopes that the hospital will do the PCR. I am even more concerned about my possible contact with her... despite the mask and gloves...

Today there were 36 patients (all by phone), with 10 consultations of patients with respiratory symptoms, contacts with patients with respiratory symptoms, and follow-up of previous cases. In these cases have been two with ageusia and anosmia: a 29-year-old man presenting with low-grade fever, ageusia and anosmia without dyspnea, cough or other symptoms, and a 40-year-old woman with afternoon fever, cough, anosmia, and dysgeusia. What is the true symptomatology of COVID-19? It is just cough, fever and dyspnea? [21,22]. 


\section{Epidemiology International Journal}

\section{March 29-Sunday}

80,110 infected, 6,803 dead, and 14,709 recovered in Spain. The Prime Minister, Pedro Sánchez stops all nonessential activity in Spain until April 9 [23].

I continue to control the temperature: I have no fever or other symptoms... but I feel strange, unreal.

\section{March 31st}

6,424 positive cases and 708 deaths in Castilla La Mancha. In Spain: 94,417 infected 8,189 dead and 19,259 recovered.

I phone the nurse who works in my office to know how it is. I hear her with a weak voice. She continues with a cough and fever; diarrhea was improved. The PCR, however, was negative... I say that it should be repeated...

Why are so many healthcare professionals infected in Spain? In Castilla la Mancha they could be between 15-30\%. The belief that this outbreak, like a Big Bang, would not occur, the lack of foresight and protective equipment, and perhaps cross-infection in healthcare facilities, mainly hospitals and nursing homes could be some of the reasons [24].

33 patients; all of these were telephone inquiries. The waiting room is empty.

Eight respiratory cases or contacts or follow-up of both: a 50-year-old male with a fever of $38^{\circ}$ since yesterday, 36-year-old man with sporadic cough, 39-year-old woman with cough, occasionally with shortness of breath (she is asthmatic), low-grade fever, holocranial headache and asthenia; her two parents COVID-19 positive (her mother at home and her father hospitalized); she was with them 15 days ago, a 42-year-old woman with odynophagia for 10 days without fever and a slight cough with expectoration, a 32-year-old man with hoarseness, but better of cough and fever, a 38-year-old man who started as back pain, and added slight cough and diarrhoea, 53-year-old woman without fever but slight nighttime cough, 68-year-old man who still has a sore throat, 58-year-old woman with little cough and possible contact with COVID-19.

Are there more cases respiratory cases in young people and in male adults? [25].

\section{Conclusion}

In the second half of March the COVID-19 outbreak broke out in my consultation. It was not a kind of chance (by lottery) with one or two patients; there was a Big Bang of respiratory infections. I drew a figure (FIGURE 1) with the percentage of respiratory infections over the total number of patients seen each day, as of February 26. Are we at the peak of the new infection curve? However, by not doing PCR for diagnosis, this increase in cases mixes real cases of COVID-19 with other aetiologies. Even the great anxiety generated in the population can confuse the symptoms. The organization of the consultation also exploded. A triage system separated respiratory patients who came in person (that they were very few) was instituted and almost all consultations were made by phone. Although "classic" consultations for chronic patients persisted (mainly repetition of prescriptions), and a few other acute problems, between $20-50 \%$ of consultations were for subjects related to COVID-19 (patients with respiratory infections without PCR performed, contacts with patients, follow-up of these, anxiety due to the epidemic situation and sick leave due to all these circumstances). How will this affect the organization of my consultation in the next weeks or months? Several nearby health professionals became ill and one died: COVID-19 carries the trace of true death, very different from its logical and abstract possibility.

\section{References}

1. López Á (2020) Science is publicized openly against the coronavirus Academia. Española de Dermatología y Venereología.

2. Turabian JL (1995) Cuadernos de Medicina de Familia y Comunitaria. Una introducción a los principios de Medicina de Familia. Notebooks of Family and Community Medicine. An introduction to the principles of Family Medicine. Madrid: Díaz de Santos.

3. Turabian JL, Perez-Franco B (2016) The Family Doctors: Images and Metaphors of the Family Doctor to Learn Family Medicine. New York, Nova Publishers.

4. Tregoning J (2020) Coronavirus diaries: hello from home. Nature.

5. Ouyang H (2020) I'm an E.R. Doctor in New York. None of Us Will Ever Be the Same. A Covid diary: This is what I saw as the pandemic engulfed our hospitals. The New York Times.

6. Bowling A (2000) Research methods in health. Investigating health and health services. Open University Press.

7. Turabian JL (2020) A family doctor's clinicalepidemiological diary in Spain of the times of covid-19. February 24 to march 15: is covid-19 a lottery? Epidemol Int J.

8. Oppenheim AN (1984) Questionnaire design and 


\section{Epidemiology International Journal}

attitude measurement. London, Heinemann.

9. Turabian JL (2020) Micro-Impact of the Pandemic by Covid-19 in the General Medicine: Clinical and Epidemiological Reflections from the Situation in Spain March 2020. Epidemol Int J 4(2): 1-11.

10. Magoon V (2020) Operationalizing Virtual Visits during a Public Health Emergency. FPM 27(3): 5-12.

11. Editorial (2020) COVID-19: protecting health-care workers. Lancet 395(10228): 922.

12. Booth TF, Kournikakis B, Bastien N, Ho J, Kobasa D, et al. (2005) Detection of Airborne Severe Acute Respiratory Syndrome (SARS) Coronavirus and Environmental Contamination in SARS Outbreak Units. Int J Infect Dis 191(9): 1472-1477.

13. Li R, Pei S, Chen B, Song Y, Zhang T, et al. (2020) Substantial undocumented infection facilitates the rapid dissemination of novel coronavirus (SARS-CoV2). Science 368(6490): 489-493.

14. Solís A (2020) Scientists suspect Covid-19 arrived in Italy six months ago. Economía Digital.

15. Brooks M (2020) COVID-19: Mental Illness the 'Inevitable' Next Pandemic?. Medscape.

16. Turabian JL (2020) Implications on mental health by the coronavirus disease 2019 (covid-19) pandemic: The role of general practitioner. Archives of Psychiatry and Mental Health.

17. Adam D (2020) Modelers Struggle to Predict the Future of the COVID-19 Pandemic. Disease experts have largely focused on how we got to where we are now with coronavirus infections. Improved data collection and sharing can enhance projections of what's to come. The Scientist.

18. Maxmen A (2020) How much is coronavirus spreading under the radar? Three leading health officials talk about gauging the size of local outbreaks, and why containment strategies aren't futile yet. Nature.

19. McBryde E (2020) The value of early transmission dynamic studies in emerging infectious diseases. Lancet Infect Dis 20: 512-513.

20. Kucharski AJ, Russell TW, Diamond C, Liu Y, Edmunds J, et al. (2020) Early dynamics of transmission and control of COVID-19: a mathematical modelling study. Lancet Infect Dis 20: 553-558.

21. Spinato G, Fabbris C, Polesel J, Cazzador D, Borsetto D, et al. (2020) Alterations in Smell or Taste in Mildly Symptomatic Outpatients With SARS-CoV-2 Infection. JAMA.

22. Otto MA (2020) Unusual Presentations of COVID-19: 'Our Ignorance Is Profound. Medscape.

23. Marcos J (2020) Paralyzed all non-essential activity in Spain. El País.

24. Turabian JL (2020) The epidemiological hypothesis of "the trojan horse": were hospitals the main vectors of the exponencial beginning of coronavirus disease 2019 (COVID-19) in Spain and other countries?. Epidemol Int J 4(3): 1-6.

25. González D (2020) USA: 38\% of those hospitalized for Covid-19 are young adults. France24. 